\title{
Editorial
}

\section{Inorganic Fullerene-Like Nanoparticles and Inorganic Nanotubes}

\author{
Reshef Tenne 1,* and Andrey N. Enyashin ${ }^{2}$ \\ 1 Department of Materials and Interfaces, Weizmann Institute, Rehovot 76100, Israel \\ 2 Institute of Solid State Chemistry RAS Pervomayskaya Str., 91, 620990 Ekaterinburg, Russia; \\ E-Mail: enyashin@ihim.uran.ru \\ * Author to whom correspondence should be addressed; E-Mail: reshef.tenne@weizmann.ac.il; \\ Tel.: +972-8-934-2394; Fax: +972-8-934-4138.
}

External Editor: Duncan Gregory

Received: 11 November 2014 / Accepted: 25 November 2014 / Published: 28 November 2014

Fullerene-like nanoparticles (inorganic fullerenes; IF) and nanotubes of inorganic layered compounds (inorganic nanotubes; INT) combine low dimensionality and nanosize, enhancing the performance of corresponding bulk counterparts in their already known applications, as well as opening new fields of their own [1]. This issue gathers articles from the diverse area of materials science and is devoted to fullerene-like nanoparticles and nanotubes of layered sulfides and boron nitride and collects the most current results obtained at the interface between fundamental research and engineering.

Arising from fortuitous lab discovery, the commercial production of inorganic hollow nanoparticles was focused on molybdenum and tungsten disulfides. Their superior solid lubrication effects have engendered intense industrial scale-up and commercialization, with sales of thousands of tons of formulated lubricants per year [2]. One of the papers of this issue describes an attempt for further rationalization and scale-up of the manufacturing of $\mathrm{WS}_{2}$ nanoparticles after gas-solid reductive sulfurization of $\mathrm{WO}_{3}$ nanoparticles in a rotary furnace [3]. This systematic study included the investigation of many reaction parameters such as precursor type, reaction temperature and time, and the reducing atmosphere. This new technique could, in the future, become a successful alternative for increasing the yield of IF production compared to the current fluidized-bed reactor.

The fullerene-like morphology of $\mathrm{MoS}_{2}$ and $\mathrm{WS}_{2}$ considerably improves the tribological properties of these compounds, pushing ahead the large-scale use of layered sulfides in machinery, aerospace and in the future also in medical industries as dry and oil-based lubricants as well as wear-resistant surface 
coatings [4]. Such applications require the deep understanding of the factors determining the mechanical and structural stability of inorganic nanoparticles under extreme conditions of high pressure or intense irradiation [5]. The study of Cook et al. explores the failure mechanisms found in $\mathrm{WS}_{2}$ IFs treated with diverse pressure loading methods [6]. The authors uncover at least two distinct fracture modes, i.e., the collapse of quasi-spherical morphology into agglomerated plate-like sheets and the delamination and exfoliation of the IF-WS 2 nanoparticles. The latter process, is accomplished also by inductively-coupled radio-frequency plasma irradiation of multiwall $\mathrm{WS}_{2}$ nanotubes, which is discussed in another paper [7] in this issue. The authors were able to control the process of layer-bylayer "undressing" of multilayered INTs and in this manner fabricate $\mathrm{WS}_{2}$ nanotubes with ultra-thin (one to three layers) walls.

The significant stability of hollow sulfide nanoparticles (IF/INT) under shock-wave propagation or irradiation suggests their potential use as fillers for impact resilient polymer or ceramic composites [8]. Apparently, such applications of $\mathrm{WS}_{2}$ or $\mathrm{MoS}_{2}$ fullerene-like particles or nanotubes may yield polymer composites with high degree of crystallinity and, consequently, with improved thermoplastic and mechanical properties, as demonstrated by Naffakh et al. [9]. However, in many cases, the adhesion between a nanoparticle and the polymer matrix is limited to the weak van der Waals interaction and could be enhanced by means of covalent bonding at the nanoparticle-polymer interface. Surface functionalization of IFs or INTs, as reported by Raichmann et al. [10], could provide stronger adhesion of nanoparticles with the polymer matrix. The emphasis in this direction was given to the non-trivial functionalization of the hydrophobic $\mathrm{WS}_{2}$ nanotubes by hydrophilic carboxyl groups, which could further stimulate the fabrication of hydrophilic polymer composites or ceramics.

Substantial progress in safe production and pioneering use of IFs and INTs has been made possible due to the comprehensive experimental research of their formation conditions, chemical activity, mechanical and electronic characteristics. However, novel and modified nanoparticles of sulfides and other compounds, such as boron nitride, can provide a much larger diversity of new materials in catalysis, electronics and electrochemistry, and their detailed characterization is still required. Computational materials science can be a valuable tool for a preliminary study of this kind of nanoparticles [11] and this issue contains examples of theoretical papers describing investigations of this type. For example, nanotubes of noble-metal chalcogenides were designed and described as stable semiconductors in theoretical work by Zibouche et al. [12]. It can guide the experimental groups in the search of fullerene-like nanoparticles and nanotubes of other compounds.

The subjects of the presented papers cover a wide range of challenges in the area of inorganic fullerene-like nanoparticles and nanotubes. However, it can include only a few comprehensive experimental and theoretical efforts, stepwise evaluating the rationalization of the synthesis, and elucidation of the stability, mechanical, electronic and adhesive properties of these nanostructures. We believe that this thematic issue can be helpful, not only for an advanced researcher to grasp with the latest developments in this field, but also to permit a beginner to gain a deeper insight into the field of inorganic fullerene-like nanoparticles and nanotubes. 


\section{References}

1. Tenne, R.; Rosentsveig, R.; Zak, A. Inorganic Nanotubes and Fullerene-Like Nanoparticles: Synthesis, Mechanical Properties, and Applications. Phys. Status Solidi A 2013, 210, 2253-2258.

2. Zak, A.; Sallacan-Ecker, L.; Margolin, A.; Feldman, Y.; Popovitz-Biro, R.; Albu-Yaron, A.; Genut, M.; Tenne, R. Scaling Up of the $\mathrm{WS}_{2}$ Nanotubes Synthesis. Fullerenes Nanotubes Carbon Nanostruct. 2010, 19, 18-26.

3. Xu, F.; Wang, N.; Chang, H.; Xia, Y.; Zhu, Y. Continuous Production of IF-WS 2 Nanoparticles by a Rotary Process. Inorganics 2014, 2, 313-333.

4. Adini, A.R.; Redlich, M.; Tenne, R. Medical Applications of Inorganic Fullerene-Like Nanoparticles. J. Mater. Chem. 2011, 21, 15121-15131.

5. Rosentsveig, R.; Gorodnev, A.; Feuerstein, N.; Friedman, H.; Zak, A.; Fleischer, N.; Tannous, J.; Dassenoy, F.; Tenne, R. Fullerene-like $\mathrm{MoS}_{2}$ Nanoparticles and Their Tribological Behavior. Tribology Lett. 2009, 36, 175-182.

6. Cook, J.; Rhyans, S.; Roncase, L.; Hobson, G.; Luhrs, C.C. Microstructural Study of IF-WS 2 Failure Modes. Inorganics 2014, 2, 377-395.

7. Brüser, V.; Popovitz-Biro, R.; Albu-Yaron, A.; Lorenz, T.; Seifert, G.; Tenne, R.; Zak, A. Single-to Triple-Wall $\mathrm{WS}_{2}$ Nanotubes Obtained by High-Power Plasma Ablation of $\mathrm{WS}_{2}$ Multiwall Nanotubes. Inorganics 2014, 2, 177-190.

8. Naffakh, M.; Díez-Pascual, A.M.; Marco, C.; Ellis, G.J.; Gómez-Fatou, M.A. Opportunities and Challenges in the Use of Inorganic Fullerene-Like Nanoparticles to Produce Advanced Polymer Nanocomposites. Prog. Polym. Sci. 2013, 38, 1163-1231.

9. Naffakh, M.; Díez-Pascual, A.M. Thermoplastic Polymer Nanocomposites Based on Inorganic Fullerene-like Nanoparticles and Inorganic Nanotubes. Inorganics 2014, 2, 291-312.

10. Raichman, D.; Strawser, D.; Lellouche, J.-P. Design of Experiments: Optimizing the Polycarboxylation/Functionalization of Tungsten Disulfide Nanotubes. Inorganics 2014, 2, 455-467.

11. Enyashin, A. Theoretical Studies of Inorganic Fullerenes and Fullerene-Like Nanoparticles. Isr. J. Chem. 2010, 50, 468-483.

12. Zibouche, N.; Kuc, A.; Miró, P.; Heine, T. Noble-Metal Chalcogenide Nanotubes. Inorganics 2014, 2, 556-564.

(C) 2014 by the authors; licensee MDPI, Basel, Switzerland. This article is an open access article distributed under the terms and conditions of the Creative Commons Attribution license (http://creativecommons.org/licenses/by/4.0/). 